\title{
CORRELAÇÃO ENTRE FORÇA MUSCULAR E CAPACIDADE FUNCIONAL EM HIPERTENSOS
}

\author{
Vitor Pontes Soares*, Adonai Ferreira Dias**, Davi Mota de Jesus**, Taís Silva Nascimento**, Vinícius \\ Cardoso Lago*, Ana Lúcia Barbosa Góes*** \\ Autor correspondente: Vitor Pontes Soares - vitorsoares11.2@bahiana.edu.br \\ * Bacharel em Fisioterapia pela Escola Bahiana de Medicina e Saúde Pública (EBMSP). \\ ** Discente do curso de Fisioterapia da Escola Bahiana de Medicina e Saúde Pública (EBMSP). \\ *** Mestre em Medicina e Saúde Humana pela Escola Bahiana de Medicina e Saúde Pública (EBMSP).
}

\begin{abstract}
Resumo
Introdução: Hipertensão é condição clínica de alta prevalência e principal fator de risco para doenças cardiovasculares e cerebrovasculares. Uma das repercussões hemodinâmicas é a diminuição do aporte energético da musculatura pelo comprometimento da perfusão, gerando redução na força muscular (FM). FM está diretamente relacionada à capacidade funcional (CF), medida que representa movimentos funcionais habituais. Objetivo: Verificar associação entre força muscular e capacidade funcional em indivíduos hipertensos. Metodologia: Estudo de corte transversal, com sujeitos hipertensos (PAS $\geq 140 \mathrm{mmHg}$ e/ou PAD $\geq 90 \mathrm{mmHg}$ ), ambos os sexos. FM foi avaliada através da Força de Preensão Palmar (FPP) com Dinamômetro Jamar®, conforme o protocolo do American Society of Hand Therapists (ASHT). A CF foi avaliada a partir da distância percorrida (DP) no teste de caminhada de seis minutos (TC6'). Para verificar associação entre FPP e DP foi utilizado o teste de correlação de Spearman, com nível de significância de $5 \%$. Este projeto foi aprovado pelo Comitê de Ética-Bahiana sob o número de protocolo 413.317, CAAE n 16952113.0000 .5544 . Resultados: Dos 29 participantes analisados, idade média foi de $47,6 \pm 7,6$ anos, sexo feminino $(72,4 \%)$, com 12 ou mais anos de estudo $(55,2 \%)$, nunca fumaram (75\%), em uso de medicação (77,80\%). PAS média foi de $125,56 \pm 18,89 \mathrm{mmHg}$ e PAD de $82,15 \pm 12,58 \mathrm{mmHg}$. A medida de correlação entre FPP e DP foi moderada $(R=0,45, p<0,05)$. Conclusão: Existe correlação positiva moderada entre a força muscular e a distância percorrida no TC6' em indivíduos hipertensos. No entanto, faz-se necessário avaliar o papel da hipertensão, idade, sexo e altura tanto na força muscular como na distância percorrida para entender melhor essa relação. Palavras-Chave: Hipertensão; Força muscular; Capacidade funcional.
\end{abstract}




\title{
CORRELATION BETWEEN MUSCLE STRENGHT \\ AND FUNCTIONAL CAPACITY IN HYPERTENSIVE INDIVIDUALS
}

\begin{abstract}
Introduction: Hypertension is a highly prevalent clinical condition that also constitutes a major risk factor in the onset of cardiovascular and cerebrovascular diseases. One of the hemodynamic effects is the reduction of energy intake by the muscles due to impaired perfusion, causing reduction in muscle strength (MS). MS is directly correlated to functional capacity (FC). Objective: To determine the association between muscle strength and functional capacity in hypertensive individuals. Methods: Cross-sectional study, hypertensive subjects (PAS $\geq 140 \mathrm{mmHg}$ and / or $\mathrm{PAD} \geq 90 \mathrm{mmHg}$ ), both sexes. FM was assessed by hold Palmar Force (PPF) with Dynamometer Jamar $®$, according to the American Society of Hand Therapists' protocol (ASHT). The FC was evaluated from the distance covered (DC) in the six-minute walk test (6MWT). To assess the association between FPP and DP the Spearman correlation test was used, with a $5 \%$ significance level. This project was approved by the BAHIANA's Ethics Committee under the number 413,317, and the CAAE number 16952113.0000.5544. Results: Among the 29 study participants, the mean age was $47.6 \pm 7.6$ years, $72.4 \%$ were female, with 12 or more years of education (55.2\%), who had never smoked (75\%), and with $77.80 \%$ of them on medication. Average PAS was $125.56 \pm 18,89 \mathrm{mmHg}$ and DBP $82.15 \pm 12,58 \mathrm{mmHg}$. The measure of correlation between FPP and RPP was moderate $(R=0.45, \mathrm{P}<0.05)$. Conclusion:There is a moderate positive correlation between muscle strength and the distance walked during the 6MWT in hypertensive individuals. However, it is necessary to assess the role of high blood pressure, age, gender and height both in muscle strength and the distance walked to better understand this interaction.
\end{abstract}

Keywords: Hypertension; Muscle strenght; Functional capacity.

\section{INTRODUÇÃO}

\begin{abstract}
A hipertensão arterial sistêmica (HAS) é uma condição clínica de alta prevalência, variando de 19 a $45 \%$ em diferentes regiões do Brasil. (1) Atinge indivíduos a partir dos 18 anos de idade, sendo apontada como um dos principais fatores de risco modificáveis para doenças cardiovasculares e cerebrovasculares, levando a óbito 7,6 milhões de pessoas no mundo por eventos secundários atribuídos. ${ }^{(2)}$ É também considerado um dos principais problemas de saúde pública, gerando aposentadoria precoce em $40 \%$ dos casos, por gerar impacto na capacidade do indivíduo em realizar suas funções. ${ }^{(3)}$
\end{abstract}

A habilidade física e mental para manter uma vida independente e autônoma, ou a plena realização de uma tarefa ou ação pelo indivíduo é definida como capacidade funcional (CF), traduzindo o potencial de realizar tarefas ou funções das atividades básicas de vida diárias. (4) $\mathrm{Na}$ literatura atual, tem sido estudada principalmente na população idosa, portadores de doenças cardíacas e respiratórias, hipertensos e mulheres. ${ }^{(5-7)}$ É possível avaliar a CF a partir de diferentes instrumentos como: testes em esteira, testes de exercício cardiopulmonar, testes de distância bidirecional, conhecido como Shuttle 
Walk Test e o teste de caminhada de seis minutos (TC6'). ${ }^{(5,7-9)}$

Um componente importante da capacidade funcional é a aptidão física relacionada a saúde (AFS), abordada como a capacidade do corpo de se adaptar a condições de determinada demanda, sem permitir que o mesmo atinja complexa exaustão, tendo as resistências cardiovascular e muscular como importantes fatores determinantes desta condição. $(4,6,10)$ Um fator que pode influenciar a aptidão física e consequentemente a CF é o grau de força muscular (FM) desenvolvido pela pessoa, ou a capacidade de exercer força/tensão para realização de movimento, uma vez que a força muscular pode estar relacionada à velocidade e qualidade na execução de movimentos. $(4,6,10)$

Estudos prévios demonstram menor força muscular e capacidade funcional em hipertensos, sugerindo que a hipertensão pode influenciar essas condições. (7) $A$ alteração da capacidade funcional em indivíduos hipertensos tem sido relacionada aos mecanismos de regulação das grandes e pequenas artérias. Ao modificar suas propriedades estruturais, se adaptando a partir de estímulos de fluxo e pressão na luz do vaso, é gerado um aumento da resistência vascular periférica, principalmente em condições de baixo fluxo, prejudicando o transporte e a permeabilidade para o aporte nutricional e energético dos músculos. ${ }^{(11)}$

O componente que parece estar mais envolvido na gênese e manutenção da hipertensão é a Resistência Vascular Periférica (RVP), e seus determinantes, como as características de contratilidade dos vasos musculares periféricos. $O$ aumento da RVP se dá por vasoconstricção e a manutenção desse padrão pode provocar hipoperfusão muscular por tempo prolongado. Se associar a essa condição, o fato de que o indivíduo em vários momentos da vida realiza atividades que aumentam a demanda metabólica e considerando que ele vive em condição restritiva, pode ser que a hipertensão seja um fator que gere diminuição do aporte de nutrientes para o músculo, com comprometimento na sua função contrátil, prejudicando a capacidade de realizar exercícios. ${ }^{(11)}$
A Força de Preensão Palmar (FPP) está relacionada a maior parte das atividades de vida diária (AVD's), não sendo uma simples medida de avaliação de força das mãos ou membros superiores. ${ }^{(12,13)}$ Sua aplicação clínica é diferenciada, pois é considerado indicador de força total do corpo e pode ser empregado em testes de aptidão física. $(9,14,15) \mathrm{O}$ que tem sido pouco estudado é a relação entre a força muscular e a capacidade funcional, e o papel da hipertensão nessa relação. São encontrados na maioria dos estudos, apenas dados descritivos sobre como se comporta cada variável nas populações estudadas, não apresentando testes para o estabelecimento de relação entre elas. (5,9,10,12)

Conhecer a relação entre força muscular e a distância percorrida se faz importante para entender de que forma a capacidade funcional pode ser in fluenciada. Desta forma o objetivo do presente estudo foi verificar a associação entre força muscular e capacidade funcional em indivíduos hipertensos.

\section{MATERIAIS E MÉTODOS}

Estudo de corte transversal, analítico, realizado de maio de 2014 a setembro de 2015, com participantes de ambos os sexos, com diagnóstico de hipertensão há pelo menos 2 meses, faixa etária de 18-60 anos, acompanhados no Ambulatório Docente Assistencial da Bahiana (ADAB) e no Complexo Comunitário Vida Plena (CCVP), vinculados à Escola Bahiana de Medicina e Saúde Pública, em uso regular de medicação anti-hipertensiva. Foram excluídos indivíduos fumantes com obesidade tipo II, doenças neurológicas, respiratórias (asma, bronquite, pneumonia recente - últimos 6 meses), alergias, alterações mentais, depressão, insuficiência renal, gravidez e Diabetes Melito associadas à HAS e história de eventos cardiovasculares prévios (infarto do miocárdio, insuficiência cardíaca, angina instável, doença arterial periférica) e problemas ortopédicos prévios, detectados em prontuário ou com diagnóstico referido.

Para avaliar a força muscular foi realizado o teste de FPP, através do dinamômetro de preensão 
palmar da marca Jamar $^{\circledR}$, o mais utilizado nos estudos e considerado padrão ouro para avaliação. (15) Foi utilizado o protocolo segundo American Society of Hand Therapists (ASHT): ${ }^{(13)}$ individuo sentado, confortável, cotovelo ao lado do corpo, fletido a $90^{\circ}$, antebraço neutro e o punho entre $0-30^{\circ} \mathrm{de}$ extensão. Avaliador sustenta o dinamômetro, cuja manopla foi fixada na posição dois, orientando a execução da avaliação iniciada pelo membro dominante, intercalando os membros por três repetições, considerada a de maior valor.

A CF pode ser medida de diversas formas, sendo o teste de caminhada de seis minutos (TC6') o mais utilizado, por se tratar de um teste válido, simples, sem necessidade de equipamentos sofisticados, nem mesmo treinamentos avançados para os avaliadores. O teste reflete as possíveis limitações nos exercícios cotidianos, sendo mais fidedigno à capacidade de realizar AVDs que as demais formas de avaliação.(7)

O TC6' foi realizado de acordo com as orientações da American Thoracic Society. ${ }^{(16)}$ Os sujeitos foram instruídos a caminhar o mais rápido possível, sem correr, em uma pista de 30 metros durante seis minutos e orientados a interromper o teste na presença de dispneia, fadiga intensa, taquicardia e/ou qualquer outra situação de desconforto de qualquer natureza. Foram proferidas apenas frases informativas no segundo e quarto minutos sobre tempo restante do teste, não podendo apresentar caráter de incentivo. Foram aferidas também frequência cardíaca (FC) e saturação de oxigênio $\left(\mathrm{SpO}_{2}\right)$ com oxímetro de pulso no mesmo período de tempo das informações, e pressão arterial (PA) com um esfigmomanômetro digital da marca Omron ®. Dois testes foram aplicados com intervalo de quinze minutos entre eles, sendo considerado para análise o teste de maior distância percorrida. Os sujeitos da pesquisa vestiram-se com roupas confortáveis e tênis para realização do teste.

Anteriormente às avaliações, os sujeitos responderam a um questionário contendo informações sociodemograficas, de hábitos de vida e saúde, e foram submetidos a avaliação de peso e estatura através de balança com estadiômetro da marca
Welmy® e pressão arterial (PA) conforme a I Diretriz Brasileira de prevenção cardiovascular. ${ }^{(2)}$ Todas as avaliações foram realizadas por um pesquisador cego e treinado para este fim.

Foram definidas para análise as seguintes variáveis: idade (anos), sexo (dicotômica), estado civil (casado/união estável, separado/divorciado, solteiro, viúvo), escolaridade (anos de estudo), subdividida em 1-4 anos, 5-8 anos, 9-11 anos e 12 ou mais anos, cor da pele (IBGE: preta, branca, parda, amarela, indígena, outra), tabagismo (nunca fumou, fumante, ex-fumante), consumo de bebida alcóolica (dicotômica), PAS $(\mathrm{mmHg}), \operatorname{PAD}(\mathrm{mmHg})$, FC (bpm). A variável independente desse estudo foi a intensidade da força de preensão palmar (Kgf) nos indivíduos hipertensos, e a variável dependente foi a distância percorrida $(\mathrm{m})$.

Foi utilizada média e desvio-padrão para análise descritiva das variáveis quantitativas idade (anos), escolaridade (anos de estudo), capacidade funcional (distância percorrida), força muscular global (Kgf) se distribuição normal e mediana e intervalo interquartil para distribuição dos dados assimétricos Variáveis categóricas cor da pele, estado civil, consumo de bebida alcoólica serão descritas em valores absolutos e em porcentagem.

O tamanho amostral foi calculado através da calculadora WinPepi®, com base num $r=0,57$ e num poder de estudo de $90 \%$.(9) Para verificar normalidade da amostra foi realizado o teste de Shapiro-Wilk com a variável de desfecho. Para verificar associação entre Força Muscular e Capacidade Funcional foi realizada correlação de Spearman, com nível de significância estatística de $5 \%$, em hipótese bicaudal. Os dados foram organizados no programa Statistical Package for Social Sciences (SPSS) 14.0 para Windows para posterior análise.

Esse projeto foi aprovado pelo Comitê de Ética em Pesquisa da Escola Bahiana de Medicina e Saúde Pública, CAAE n 16952113.0000.5544, de acordo com a resolução 466/12 do Conselho Nacional de Saúde. A coleta iniciou após informação sobre a pesquisa ao participante e assinatura de termo de consentimento livre e esclarecido. 


\section{RESULTADOS}

A amostra foi composta por 29 participantes com

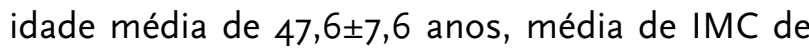

$29,2 \pm 3,7 \mathrm{Kg} / \mathrm{m}^{2}$, predominância do sexo feminino $(72,4 \%)$, casada $(58,6 \%)$, com bom nível de escolaridade com 12 ou mais anos de estudo $(55,2 \%)$, cor da pele preta $(58,6 \%)$. (Tabela 1 )

Tabela 1 - Características sociodemográficas de indivíduos hipertensos Salvador-Ba. 2015. (n=29)

\begin{tabular}{|c|c|}
\hline VARIÁVEIS & MEDIA FFLDP \\
\hline Idade (anos) & $47,6 \pm 7,6$ \\
\hline IMC (Kg/m) & $29,2 \pm 3,7$ \\
\hline Sexo & $n(\%)$ \\
\hline Feminino & $21(72,4)$ \\
\hline \multicolumn{2}{|l|}{ Estado Civil } \\
\hline Casado/união estável & $17(58,6)$ \\
\hline Solteiro & $9(31,0)$ \\
\hline Separado/divorciado & $2(6,9)$ \\
\hline Viúvo & $1(3,4)$ \\
\hline \multicolumn{2}{|l|}{ Anos de Estudo (anos) } \\
\hline Até 4 & $2(6,9)$ \\
\hline $5-8$ & $4(13,8)$ \\
\hline $9-11$ & $7(24,1)$ \\
\hline 12 ou mais & $16(55,2)$ \\
\hline \multicolumn{2}{|l|}{ Cor da Pele (IBGE) } \\
\hline Preta & $17(58,6)$ \\
\hline Parda & $10(34,5)$ \\
\hline Branca & $2(6,9)$ \\
\hline
\end{tabular}

Dos 29 participantes, a maioria não adiciona sal após refeição pronta (92,1\%), nunca fumou (79,3\%), não consome bebida alcóolica $(65,5 \%)$, em uso de medicamento regularmente $(75,9 \%)$ e está com a pressão controlada $(58,6 \%)$. A força muscular do membro dominante teve média de $30,93 \pm 10,6$, a média de força no membro não dominante foi de $29,6 \pm 9,0$ e a mediana da distância percorrida foi de 517,5 (IIQ25-75:481-562). (Tabela 2)

Tabela 2 - Características da amostra quanto aos dados de hábitos de vida e de saúde. Salvador-Ba. 2015. (n=29)

\begin{tabular}{ll}
\hline VARIÁVEIS & $\mathrm{N}(\%)$ \\
\hline
\end{tabular}

\section{Adiciona sal à refeição pronta}

Não

As vezes

Tabagismo

Nunca

Ex-Fumante

$6(20,7)$ 
Tabela 2 - Características da amostra quanto aos dados de hábitos de vida e de saúde. Salvador-Ba. 2015. (n=29)

(conclusão)

VARIÁVEIS $\quad \mathrm{N}(\%)$

\section{Consumo de bebida alcoólica}

Não

$19(65,5)$

Uso de medicamento

Sim

$22(75,9)$

Classificação da Pressão Arterial

Hipertensão Controlada

$17(58,6)$

FMD (Kgf)

$30,93 \mathrm{ffl} 110,6^{*}$

FMND (Kgf)

$29,6 \mathrm{ffl} 9, \mathrm{O}^{*}$

$\mathrm{DP}(\mathrm{m})$

FMD=Força membro dominante; FMND= Força membro não dominante; DP=Distância percorrida. *=MédiaffDP. **=Mediana [IIQ25-75] .

A correlação entre força muscular e distância percorrida foi considerada moderada, tanto para o membro dominante como para o não dominan- te, sendo estatisticamente significante. $(r=0,45$, $\mathrm{p}=0,015 ; \mathrm{r}=0,40, \mathrm{p}=0,031$, respectivamente) (Gráfico 1).

Gráfico 1 - Correlação entre força muscular e distância percorrida. Força muscular membro dominante: $r=0,45$, $p=0,015$; Força muscular membro não dominante: $r=0,40, p=0,031$
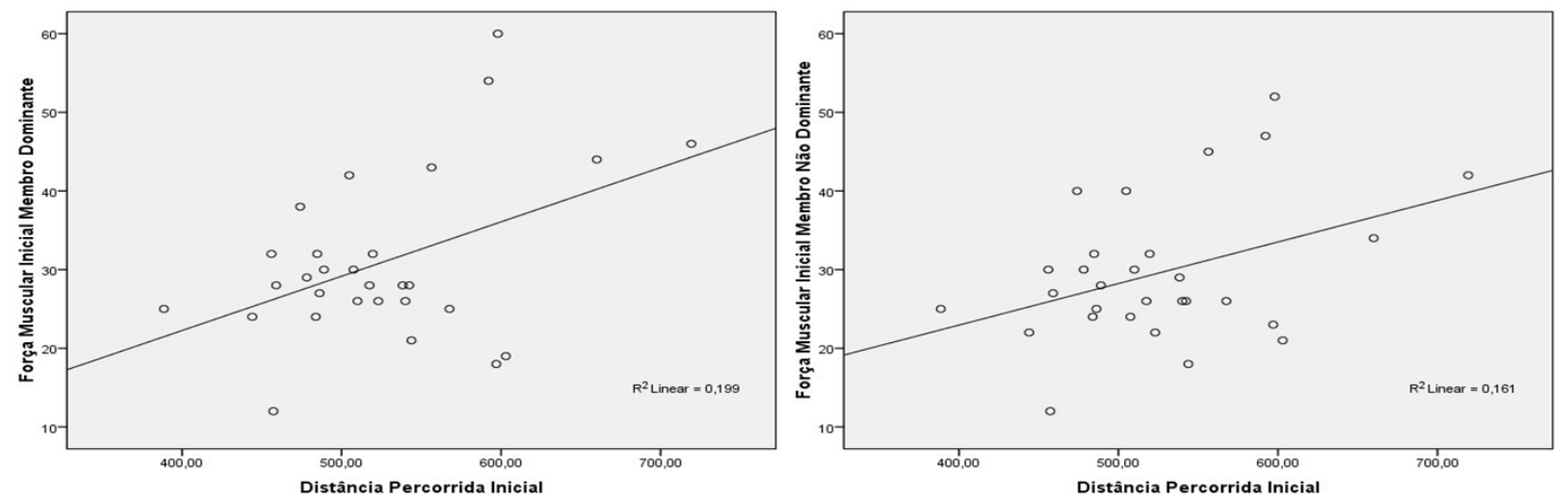

Considerando que idade e altura são variáveis que interferem no desfecho distância percorrida, foi feita a correlação entre essas variáveis, encone distância percorrida $(r=-0,46, p=0,012)$ e correlação positiva e moderada entre altura e distância percorrida $(r=0,53, p=0,003)$ (Gráfico 2). 
Gráfico 2 - Correlação entre variáveis independentes idade e altura com distância percorrida. Idade: r=-0,46, p=0,012; Altura: $r=0,53, p=0,003$

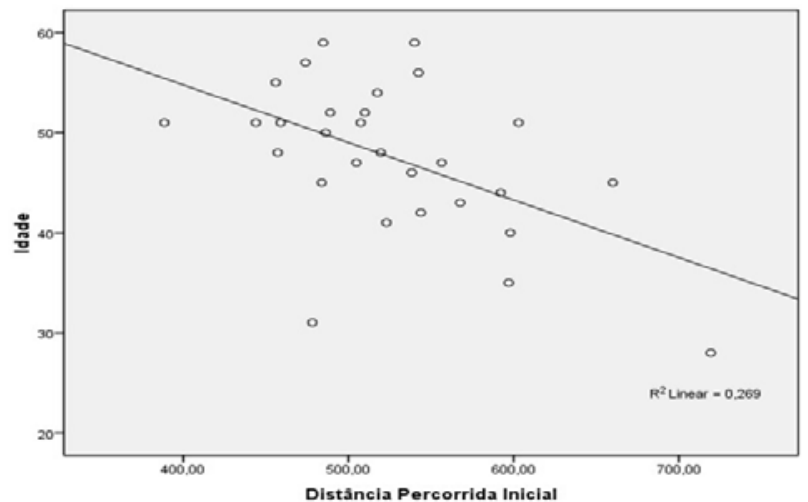

\section{DISCUSSÃO}

Esta pesquisa teve como objetivo verificar correlação entre força muscular e capacidade funcional em indivíduos hipertensos, apresentando resultados compatíveis com a literatura. Neste estudo observou-se correlação positiva moderada entre a FPP e a distância percorrida, seguindo a tendência do estudo de Pereira et al, ${ }^{(6)}$ que demonstra forte correlação $(r=0,872)$ na mesma relação em indivíduos com Doença Arterial Obstrutiva Periférica (DAOP). A diferença da força de associação entre os estudos, pode se explicar pelo mecanismo de cada afeç̧ão. Na DAOP existe um elemento de resistência vascular periférica diferente do mecanismo da HAS, em que a primeira apresenta resistência imposta por rigidez vascular irreversível e progressiva e a vasoconstricção pela HAS é secundária à hiperestimulação do sistema nervoso simpático, ${ }^{(17)}$ o que permite adaptações e possivelmente uma melhor perfusão.

Outro fator que diferencia os estudos e que pode explicar a diferença na magnitude da correlação é o instrumento de avaliação utilizado nos estudos. O presente estudo utilizou o TC6', teste mais fidedigno à capacidade funcional real, já que permite paradas durante o teste, gerando um esforço cardiovascular e muscular compatíveis, portanto, gerando um resultado com relação mais íntima com a musculatura. ${ }^{(7)}$ Já o Shuttle Walk Test tem maior

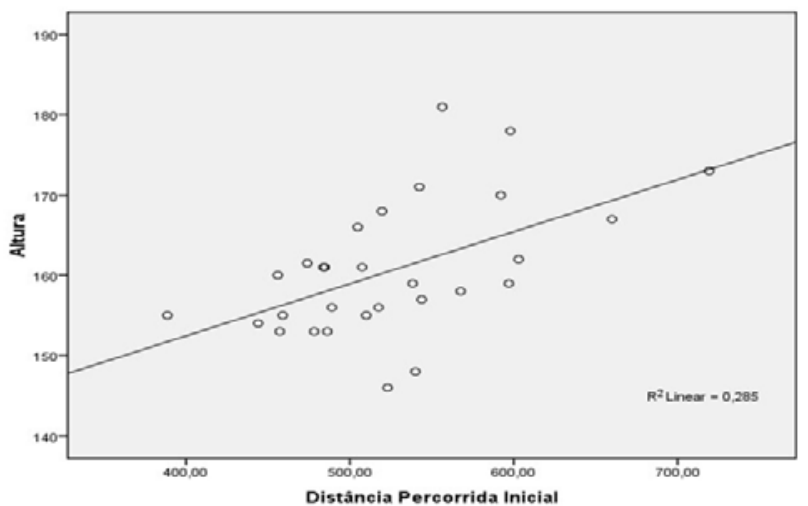

foco na avaliação do condicionamento cardiovascular e respiratório, ${ }^{(8)}$ pois é um teste de intensidade progressiva, podendo gerar confusão sobre qual variável estaria de fato influenciando no resultado, se a condição cardiovascular e respiratória ou a condição musculoesquelética.

Outras diferenças entre os estudos são o instrumento e o local de aplicação do instrumento. No presente estudo, a força de preensão manual foi eleita por se tratar de uma avaliação focada em musculatura que envolve funcionalidade e por ser largamente utilizada como avaliador de aptidão física. Já o estudo de Pereira et al, ${ }^{(6)}$ avaliou a força muscular específica de membros inferiores, utilizando como instrumento de avaliação 1 RM. Apesar de avaliar força de membros inferiores, apontada na literatura como tendo maior correlação para a capacidade funcional, é a força de preensão palmar que está melhor relacionada com a capacidade funcional.

Um estudo realizado com idosos institucionalizados apresentou correlação moderada positiva $(r=0,4)$ entre força muscular e capacidade funcional. Foram utilizados os mesmos instrumentos do presente estudo, encontrando medidas menores nas variáveis, devido as mudanças provenientes do envelhecimento. Desta forma, reforça a ideia de que existe associação entre força muscular e distância percorrida no TC6', (9) corroborando com outro estudo que apresenta correlação entre a força de preen- 
são palmar com a medida de $\mathrm{VO}_{2}$ máximo, medida utilizada como marcador de aptidão física. ${ }^{(14)}$

Um estudo de Reis et al, (5) sugere não haver relação entre a força muscular e a capacidade funcional. A população do estudo foi de mulheres na menopausa, com média de idade $44,11 \pm 15,00$. A justificativa da ausência de associação foi a queda de estrogênio, provocando sarcopenia nessa faixa etária. Um estudo confirma este achado, e demonstra que mesmo a reposição hormonal não é capaz de otimizar o desempenho muscular.(18) $\mathrm{O}$ fato do presente estudo ter a maioria mulheres, e em faixa etária semelhante, pode ter influenciado na força da correlação.

Os valores de força muscular e distância percorrida podem ser considerados abaixo da média. Em estudo de revisão, considerando várias populações, idades e sexo, a força muscular, de forma geral, foi diferente entre o sexo e a idade. Os homens obtiveram maiores valores de força de preensão palmar, em torno de 45-50Kgf, do que as mulheres, em torno de $25-30 \mathrm{Kgf}$, para todas as idades e entre as mãos dominantes e não dominante. Ainda para a idade, parece que a partir da $4^{\text {a }}$ década de vida, existe um declínio da força muscular nas mulheres. (15) Como o presente estudo obteve maior número de mulheres, essa condição também pode ter favorecido queda no valor médio da FM. O mesmo pode ser sugerido para a população hipertensa, que pode comprometer a perfusão muscular como já explicado. ${ }^{(11)}$

A distância percorrida obteve padrão de comportamento semelhante ao da força muscular, em que, de forma geral, foi diferente para sexo. Os homens obtiveram maiores valores de distância percorrida - aproximadamente 570 metros - do que as muIheres, com valores em torno de 530 metros. ${ }^{\left({ }^{(9)}\right)}$ Assim como na FM, a maioria do sexo feminino e a hipertensão podem ter favorecido queda no valor médio da DP.

Em um estudo realizado no Brasil, a frequência de hipertensão foi maior em mulheres, aumentou com a idade, diminuiu com a escolaridade, foi maior em indivíduos com cor de pele preta e viú- vos. Após ajuste para fatores de confusão, a frequência de hipertensão foi maior em indivíduos com excesso de peso e diabetes. ${ }^{(20,21,22)}$

É comum encontrar nos estudos epidemiológicos, frequência de HAS maior em homens. ${ }^{(21,23)} \mathrm{No}$ entanto, devido aos locais de captação serem em instituições de assistência, atualmente o número maior de mulheres foi esperado devido à maior procura desta população por serviços de saúde.(20) Não foi possível observar no presente estudo a relação entre idade e HAS, nem entre níveis de escolaridade e HAS, porque houve um controle rígido da idade para que a amostra fosse homogênea, e a maioria dos participantes apresentava alto nível de escolaridade $(55,2 \%$ com 12 ou mais anos de estudo).

A maior frequência de HAS em pessoas de cor de pele preta pode estar relacionada à predisposição genética, já discutida na literatura ${ }^{(24)}$ e o excesso de peso pode estar relacionado à renda, que não foi avaliada neste estudo, bem como aos hábitos de alimentação. Um estudo observou que em mulheres com sobrepeso e obesas, de baixo nível socioeconômico, existia baixa ingestão de energia diária total, com ingesta ligeiramente elevada de proteínas, elevado consumo de ácidos graxos saturados e baixa ingestão de fibras. ${ }^{(25)}$

Quanto aos hábitos de vida e saúde, a maioria não adiciona sal após refeição pronta, nunca fumou, relata não consumir bebida alcóolica, está em uso de medicação e a pressão está controlada. Um estudo encontrou dados semelhantes e relacionou as mudanças nos hábitos de vida e saúde com a melhora na comunicação sobre a importância da mudança dos hábitos de vida para minimizar a HAS e outros eventos cardiovasculares. ${ }^{(20)}$

Este estudo teve como limitações o rígido controle dos critérios de inclusão, diminuindo a validade externa desses dados. A hipertensão, idade, sexo e altura podem ser fatores intervenientes dessa associação, sendo necessária realização de análise de regressão, não sendo possível sua realização nesse momento devido ao número amostral insuficiente. 


\section{CONCLUSÃO}

Existe correlação positiva moderada entre a força muscular e a distância percorrida em indivíduos hipertensos. No entanto, faz-se necessário avaliar o papel da hipertensão, idade, sexo e altura tanto na força muscular como na distância percorrida para entender melhor essa relação.

\section{REFERÊNCIAS}

1. Passos VMDA, Assis TD, Barreto SM. Hipertensão arterial no Brasil: estimativa de prevalência a partir de estudos de base populacional. Epidemiol serv. saúde. 2006;15:35-45.

2. Sociedade Brasileira de Cardiologia. I Diretriz Brasileira de prevenção cardiovascular. Arq. bras. cardiol. 2013;101:78.

3. Kohlmann Jr. O, Guimarães AC, Carvalho MHC, Chaves Jr. HC, Machado CA, Praxedes JN et al. III Consenso Brasileiro de Hipertensão Arterial. Arq. bras. endocrinol. metabol. 1999;43:257-286.

4. Araujo DSMS, Araújo CGS. Aptidão física, saúde e qualidade de vida relacionada à saúde em adultos. Rev. bras. med. esporte. 2000;6:194-203.

5. Reis JG, Costa GC, Schmidt A, Ferreira CHJ, Abreu DCC. Do muscle strengthening exercises improve performance in the 6-minute walk test in postmenopausal women? Rev. bras. fisioter. 2012;16:236-240.

6. Pereira DAG, Faria BMA, Gonçalves RAM, Carvalho VBF, Prata KO, Saraiva OS et al. Relação entre força muscular e capacidade funcional em pacientes com doença arterial obstrutiva periférica: um estudo piloto. J. vasc. bras. 2011;10:26-30.

7. Ramos R, Ferreira AS. Capacidade funcional de adultos com hipertensão avaliada pelo teste de caminhada de seis minutos: revisão sistemática. Fisioter. pesqui. 2014;21:257-263.

8. Lima AGD, Marques A, Felizardo I, Toro C. Valores de referência para o teste de caminhada com carga progressiva em indivíduos saudáveis: da distância percorrida às respostas fisiológicas. J. bras. pneumol. 2013;39:190-197.

9. Reis FIRL, Navega MT, Quitério RJ, Ambrozin ARP. Existe Correlação Entre Força Muscular com Teste de Caminhada de Seis Minutos em Idosos Institucionalizados? Uma ética para quantos? Revista Inspirar: movimento \& saúde. 2012;4:26-30.

10. Garcia PA, Dias JMD, Dias RC, Santos P, Zampa CC. Estudo da relação entre função muscular, mobilidade funcional e nível de atividade física em idosos comunitários. Rev. bras. fisioter. 2011;15:15-22.

11. Tsioufis C, Dimitriadis K, Katsiki N, Tousoulis D. Microcirculation in Hypertension: An Update on Clinical Significance and Therapy. Curr. vasc. pharmacol [Internet]. 2015:413-417. Avaliable from: <http://www.ncbi.nlm.nih.gov/ pubmed/25659075

12. Cardoso FDS, Curtolo M, Natour J, Lombardi Júnior I. Avaliação da qualidade de vida, força muscular e capacidade funcional em mulheres com fibromialgia. Rev. bras. reumatol. 2011;51:344-350.

13. Dias JA, Ovando AC, Külkamp W, Junior NGB. Força de preensão palmar: métodos de avaliação e fatores que influenciam a medida. Rev. bras. cineantropom. desempenho hum. 2012;12:209-216.

14. Tarso P, Müller G, Alberto C, Viegas A, Armando L, Patusco P. Força muscular como determinante de eficiência do consumo de oxigênio e da máxima resposta metabólica ao exercício em pacientes com DPOC leve/ moderada*. Rev. bras. pneumol. 2012;38:541549.

15. Nascimento MF, Benassi R, Caboclo FD, Salvador ACS, Gonçalves LCO. Valores de referência de força de preensão manual em ambos os gêneros e diferentes grupos etários. Um estudo de revisão. EFDeportes.com, Revista Digital. Buenos Aires. 2010;15(151). Disponível em: http://www.efdeportes.com/efd151/forca-depreensao-manual-em-ambos-os-generos.htm

16. Wanderley FAC, Oliveira J, Mota J, Carvalho MJ. Six-minute walk distance (6MWD) is associated with body fat, systolic blood pressure, and rate-pressure product in 
community dwelling elderly subjects. Arch. gerontol. geriatr. 2011;52:206-10.

17. Bruno RM, Ghiadoni L, Seravalle G, Del'Oro R, Taddei S, Grassi G. Sympathetic regulation of vascular function in health and disease. Front. Physiol. 2012;3:1- 15.

18. Greising SM, Baltgalvis KA, Lowe DA, Warren GL. Hormone Therapy and Skeletal Muscle Strength: A Meta-Analysis. J. gerontol. 2009:1071-1081.

19. Soares MR, Alberto C, Pereira DC. Teste de Caminhada de seis minutos: valores de referência para adultos saudáveis no Brasil. J. bras. pneumol. 2011;37:576-583.

2O. Gouvea SR, Moura EC, Malta DC, Sarno F. Freqüência de hipertensão arterial e fatores associados: Brasil, 2006. Rev. saúde pública. 2009;43:98-106.
21. Rosário TM, Scala LCN, França GVA, Pereira MRG, Jardim PCBV. Prevalence, control and treatment of arterial hypertension in Nobres MT. Arq. bras. cardiol. 2009;93:622-628.

22. Ferreira SRG, Moura EC, Malta DC, Sarno F. Frequency of arterial hypertension and associated factors: Brazil, 2006. Rev. saúde publica. 2009;43:98-106.

23. Kearney PM, Welton M, Reynolds K, Munter P, Whelton PK, HeJ. Global burden of hypertension: Analysis of worldwide data. Lancet. 2005;365:217-23.

24. Piccini RX, Victora CG. Hipertensão arterial sistêmica em área urbana no sul do Brasil: prevalência e fatores de risco. Rev. saúde pública. 1994;28:261-7.

25. Ferraz I, Ladeia AM, Goes P, Olivieri L, Lima ML, Guimarães AC. Calorie and Nutrient Intake in Obese Women With Low-Income. J. endocrinol. metab. 2013;3:81-90. 